\title{
Electrical Properties of Enamel Wire Insulation
}

\author{
ASIWE Uchechukwu'1, Edema Anthony², Edeafeadhe Godspower ${ }^{2}$
}

\author{
${ }^{1}$ Electrical \& Electronic Engineering Department, ${ }^{2}$ Mechanical Engineering Department \\ 1,2Delta State Polytechnic Otefe-Oghara, Delta State, Nigeria
}

\begin{abstract}
Adjustable speed drives for rotating machines have become increasingly popular as they provide possibilities of smooth and accurate process control as well as for energy savings. In such systems, due to the fact that the voltage applied at terminals of motor windings is no longer purely sinusoidal but characterized by a high content of harmonics, the appearing electrical and thermal stresses yield premature failures of the winding insulation, mainly because of an increased partial discharge (PD) activity. To tackle the problem effectively, PD-resistant enamels have been developed, by introducing various inorganic fillers into the base polymer of the wire insulation, that exhibit an increased resistance to PD activity. The effect seems, in most of the cases, to be strongly dependent on the dielectric properties of the filler used and the degree of interactions with the host materials. To elucidate the effect of material properties on the PD behavior, the presented in this thesis study concentrates on electrical characterizations of the new enamel insulation by measuring and analyzing its electric conductivity and complex dielectric permittivity and comparing these parameters with of the properties of enamel without the filler. The performed analyses show that the addition of filler results in a highly conductive and dispersive material, as compared to the base enamel. As a consequence, the top coating layer of the enamel wire insulation activates suppression of PD activity, while the insulating properties of the bulk material are kept unchanged.. The material conductivity is increased because of enhancement of charge carrier mobility as well as a shift of the maximum of trap density distributions towards lower energy levels.
\end{abstract}

Keywords: enamel wire insulation, dc conductivity, dielectric permittivity, partial discharge

\section{INTRODUCTION}

Adjustable speed drives have gained a widespread application due to a number of provided technical benefits. In particular, the use of fast switching power electronics allows for saving significant amount of energy. However, the applied voltages containing high frequency harmonics put winding insulation of electric motors at risk of failure. The continuous exposure of insulation systems to the distorted voltages increases the risk of enhanced partial discharge (PD) activity and space charge accumulation, thus causing accelerated aging and premature failure [Fabiani and Montanari, 2001], [Haq, et al, 2007]

An enamel insulation containing an unfilled base coating and micro-filled top coating has been compared to a conventional insulation [Bjorklund, and Paloniemi 1995]. The dielectric properties of filled enamel were experimentally studied in a wide range of temperature and were compared with that of conventional enamel. Since the former also contributes to the material PD resistance, the synergy effect is anticipated for the newly developed enamel insulation. The performed accelerated aging tests at high temperatures are expected to reveal that the life time of such insulation is substantially increased as compared to both the conventional. [Paulsson, et al 2013] The work focus on fundamental electrical properties of the enamel insulation, the effect of the filler on conduction and polarization mechanisms in the materials is analyzed utilizing experimental results on volume conductivity and dielectric permittivity obtained at different temperatures. Finally, PD behavior of the materials is studied and correlated with the obtained dielectric properties.

An enameled wire with conventional insulation has been used as a reference in the study.

\section{Properties of enamels}

Enamel wire insulation usually has a multilayered structure that consists of at least of two layers, a base coat and a top coat. The materials used for creating these layers are usually polyester-imide (PEI) for the former and polyamide-imide (PAI) for the latter. They are both high temperature materials with stable thermal properties. Chemical structure of the repeating unit of PAI is shown in Figure 1. For both the materials, the glass transition temperature $\left(T_{g}\right)$ is approximately $270{ }^{\circ} \mathrm{C}$ [Diaham and Locatelli, 2013], Dolu and S. Maiti, 1985]

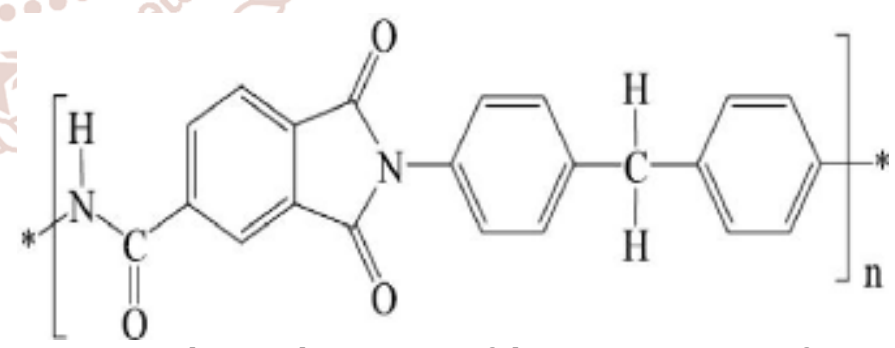

Figure1. Chemical structure of the repeating unit of PAI (Dolui S.K, D. Pal, and S. Maiti,(1985)

\section{Experiments with enameled wire samples}

Two types of enameled wires, one having a conventional and the other a filled insulation, were used in experiments. Their cross sections are illustrated in Figure 2.0.
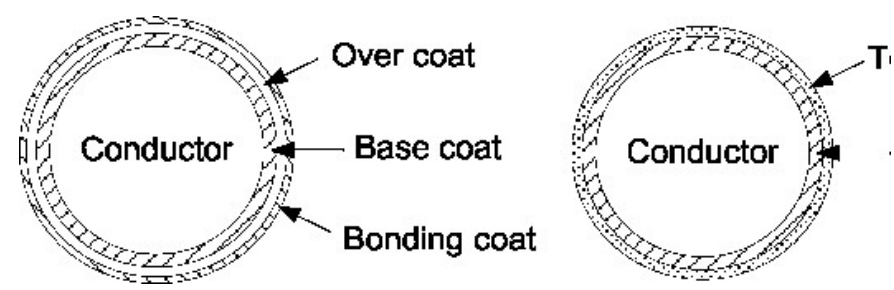

Figure2. Cross sections of conventional (a) and filled (b) enameled wires 
The conventional enamel insulation had three layers (a PEI base coat, a PAI over coat, and a modified aromatic Pcoammeter polyamide (PA) bonding coat), whereas the filled enamel had two layers (a PEI base coat and a PAI top coat). The top coat of the latter was filled by $26 \mathrm{wt} \%$ (equivalent to 8.7 vol\%) of chromium oxide microparticles, whose size varied between 0.2 and $2 \mu \mathrm{m}$ (the average size was $0.74 \mu \mathrm{m}$. The diameter of the wires in both cases was $1.18 \mathrm{~mm}$, whereas the insulation thickness was respectively 50 and $40 \mu \mathrm{m}$ for the conventional and the filled enamel layers.

The volume conductivity of the enamel insulations was measured by the standard method using a Keithley Electrometer 6517A. A three-electrode system was designed, in which the high voltage was supplied to the wire conductor, whereas the measuring and the guard electrodes were made of conductive copper tapes attached to the external enamel layer (Figure 3.0). The measuring and the guard electrodes were $4 \mathrm{~cm}$ and $1 \mathrm{~cm}$ in length, respectively. The electrode arrangement was placed in a metallic grounded box, which also allowed for controlling temperature and humidity during the measurements. In particular, tests were performed at room temperature (22$24^{\circ} \mathrm{C}$ ), and at 40,60 and $80^{\circ} \mathrm{C}$, which were achieved by using a heater and a thermostat. On the other hand, the relative humidity was maintained in the range of $30-40 \%$ by a saturated salt solution. The applied voltage was set at $100 \mathrm{~V}$, corresponding to the electric stress of approximately $106 \mathrm{~V} / \mathrm{m}$. The resulting volume conductivity $\sigma$ was determined as

$$
\sigma=\frac{I \cdot d}{U_{n} A}
$$

where $I$ is the measured current, $U$ is the applied voltage, $A$ is the area of the measuring electrode and $d$ is the insulation thickness.

The dielectric spectroscopy measurements were carried out in the frequency range from $1 \mathrm{mHz}$ to $1 \mathrm{kHz}$ by means of an Insulation Diagnostic System IDAX 300. The shielding box was also employed here for reducing the influence of parasitic capacitances between the sample and the ground potential on the measured capacitance. In this case, the external electrodes on wire samples were created by depositing thin layers of silver paint for eliminating the influence of series capacitances on the determination of permittivity and the glossy glue layer in the conductive tape. Temperature and humidity were maintained during the measurements in a similar range as for the conductivity measurements. Thermal equilibrium inside the shielding box was achieved by keeping it in an oven for 2 hours. Thereafter the experiments were carried out by applying the ac voltage of $100 \mathrm{Vrms}$. The complex permittivity of a sample was obtained by dividing the measured complex capacitance by the geometric capacitance of the samples.

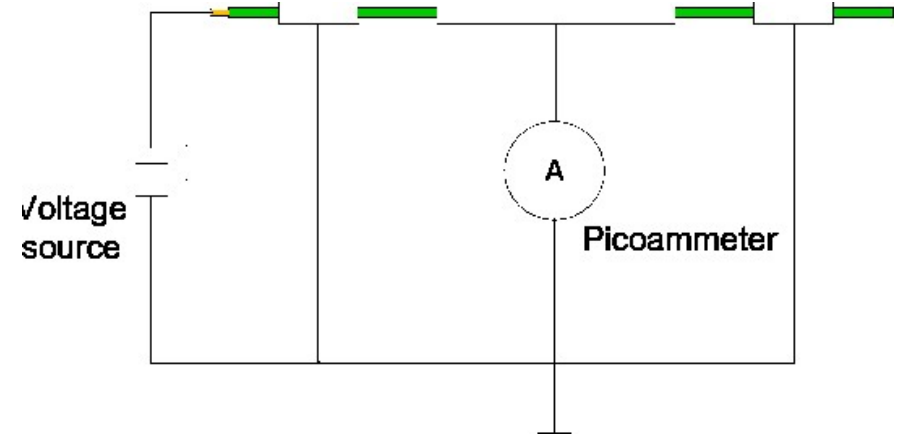

Figure3. Circuit for measuring volume conductivity of enamel coating

\section{Experimental Result on Electrical conductivity}

Typical measured volume currents for both types of the enamels are shown in Figure 4.0. For the sake of clarity, only the results obtained at the lowest $\left(22^{\circ} \mathrm{C}\right)$ and the highest temperatures $\left(80^{\circ} \mathrm{C}\right)$ are presented. It was observed that the measured currents decayed with time. They were practically independent of the material type and temperature within the first $50 \mathrm{~s}$. This could be explained by the dominance of a capacitive current during the initial stage, governed by similar capacitances of the two types of samples. After $\sim 1000$ s however, the measured currents were significantly different and the temperature effect was pronounced. Thus, the current increased by one order of magnitude as the temperature rose from $22^{\circ} \mathrm{C}$ to $80^{\circ} \mathrm{C}$ for both types of the insulation. To find conductivity values, the conductive current component has to be determined and it can only be achieved after a long measuring time when the capacitive component of the current ceases. As can be seen in this particular case, the current decayed even after $10^{5} \mathrm{~s}$ ( $\sim 28$ hours) and its purely conductive level was hardly achievable. Therefore, the measurements were stopped after $10^{5} \mathrm{~s}$ for practical reasons and the conductivities were calculated from the corresponding current magnitudes.

As seen in Figure 3.0, the obtained volume conductivities for both the enamel insulations are in general lower than the values obtained with flat samples (Table 1.0) and obey Arrhenius relation:

Table2.1. Volume conductivity of PAI resin

\begin{tabular}{c|c|c|}
\hline Material & $\begin{array}{c}\text { Electric field, } \\
\text { V/m }\end{array}$ & $\begin{array}{c}\text { Volume conductivity, } \\
\mathrm{S} / \mathrm{m}\end{array}$ \\
\hline PAI & $1.05 \times 10^{6}$ & $3.9 \times 10^{-15}$ \\
\hline \\
$\sigma(\mathrm{T})=\sigma_{0} \exp \left(-\frac{W \varepsilon}{K T}\right)$
\end{tabular}

where $\sigma_{0}$ is a constant parameter, $W_{c}$ is the activation energy for the conduction, $k$ is the Boltzmann constant, $T$ is the temperature. One may notice that the volume conductivity of the two materials is extremely low, but still higher for the filled enamel than for the conventional one. The calculated activation energies are $0.33 \mathrm{eV}$ and $0.46 \mathrm{eV}$ for the conventional and the filled enamels, respectively.

The conventional enamel coating used in this study can be considered as a series connection of three resistors corresponding to three layers in its structure. The measured volume conductivity of the conventional enamel is around two orders of magnitude lower than that of PAI obtained. For the other two materials, the parameter is 
unknown, but one can expect PEI to be the most resistive, while modified aromatic PA as the most conductive among the three constituents of the considered enamel. Thus, the resistance of three resistors in series will be determined by that of the PEI base coat. In other words, the volume conductivity of PEI should be close to that of the conventional enamel $\left(\sim 10^{-17} \mathrm{~S} / \mathrm{m}\right)$, while PAI and aromatic PA are characterized by a volume conductivity $\sigma \geq 10^{-15} \mathrm{~S} / \mathrm{m}$. The reason is that the filler modifies the property of the PAI top layer only, whereas the resistive PEI base coat remains unchanged.

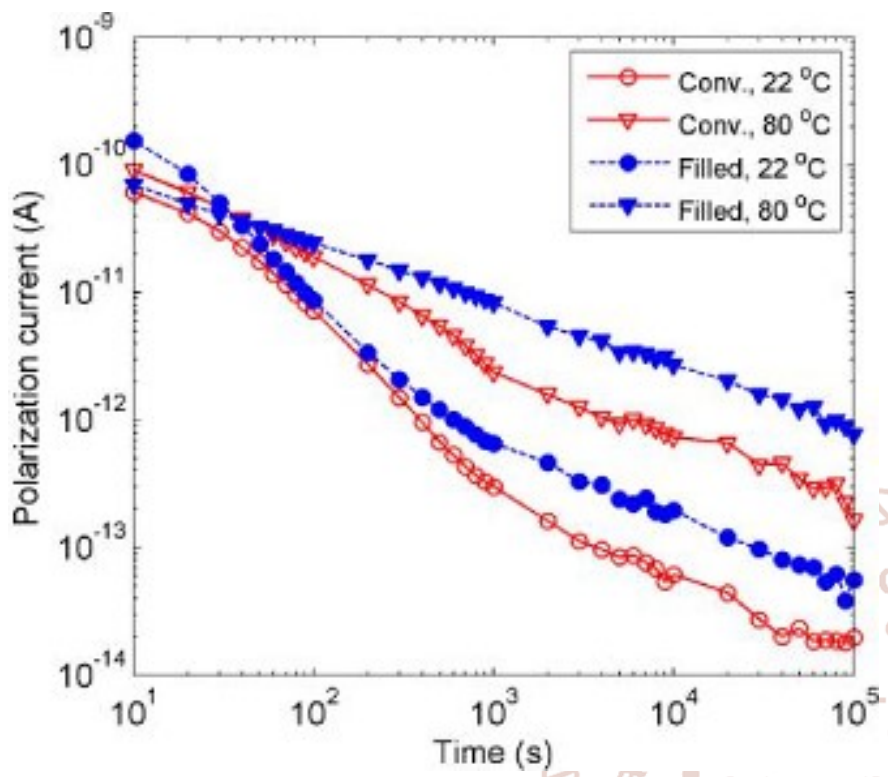

Figure4: Time variations of polarization currents of ationa conventional and filled enamels.

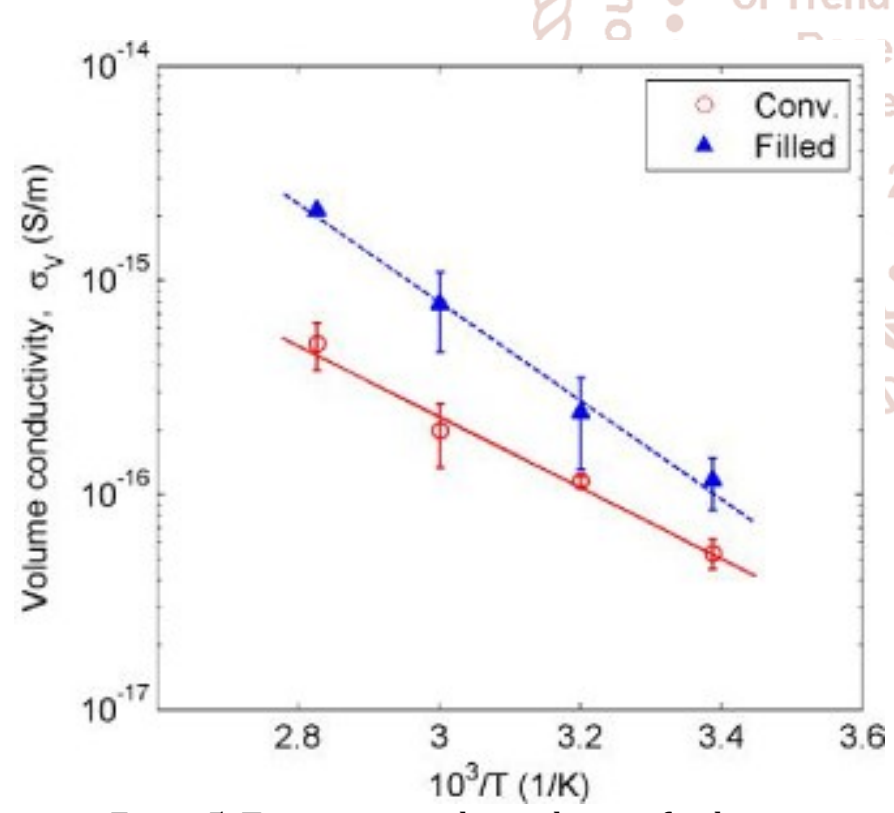

Figure5. Temperature dependence of volume conductivity of conventional and filled enamels

The conductivity has been calculated based on current value at $10^{5} \mathrm{~s}$ after test voltage application.

This procedure provided a value $7.210^{-8} \mathrm{~S} / \mathrm{m}$ that is in agreement with the measured results. This suggests

\section{Experimental Result on Complex permittivity of enamels}

Real and imaginary parts of the complex permittivity measured at various temperatures are shown in Figures 6.0 and 7.0 for the conventional and filled enamels, respectively. As it is seen, at frequencies below $10 \mathrm{~Hz}$, the imaginary part (dielectric loss) increased more rapidly with reducing frequency, whereas the real part showed a gradual rise in the whole frequency range. On the other hand, temperature affected those parameters differently: while the temperature rise reduced the real part at frequencies above $1 \mathrm{~Hz}$, it considerably increased the imaginary part. It should be noted that coefficients of thermal expansion are low for both polymeric materials (less than $410-5 \mathrm{~K}^{-1}$ [Ma, et al, 2010], Hasegawa, et al, 2010.] and for copper conductor ( 1.710-5 K-1 [19]), thus causing the thermal expansion less than $0.3 \%$ for the enamel layers and the wire conductor. Apparently, this could not account for the decrement of the real part of the complex permittivity, which was as high as $10 \%$ when the temperature increased from 24 to $100{ }^{\circ} \mathrm{C}$. Localized maxima were observed in the loss curves of conventional enamel (Figure 6.0b). They shifted towards higher frequencies and became more considerable as temperature rose. However, such localized peaks were not observed in the loss curves for filled enamel (Figure 7.0b). In general, the magnitudes of the real and imaginary parts of the complex permittivity were higher for the filled enamel than for the conventional counterpart that can be attributed to the influence of highly dispersive.
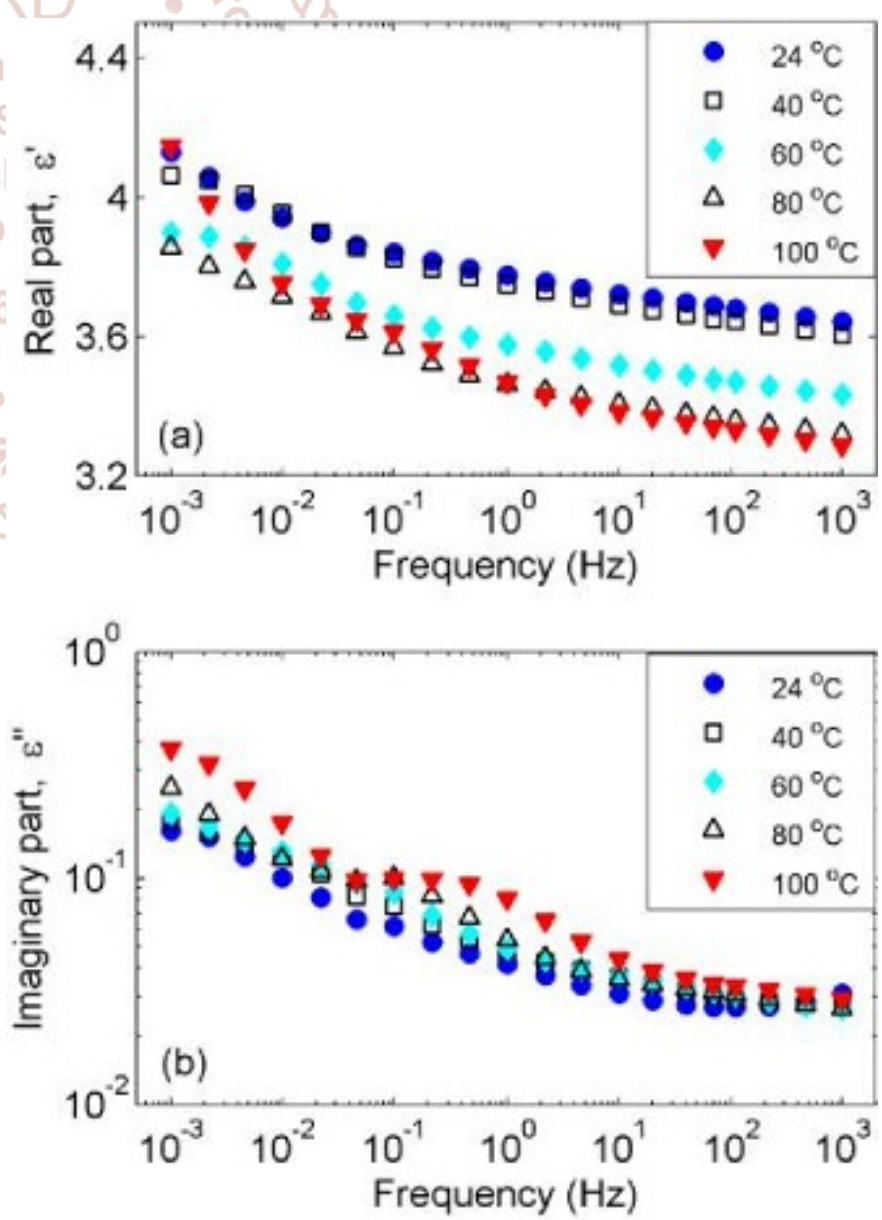

Figure6. Real (a) and imaginary (b) parts of the complex permittivity of conventional enamel at different temperatures 

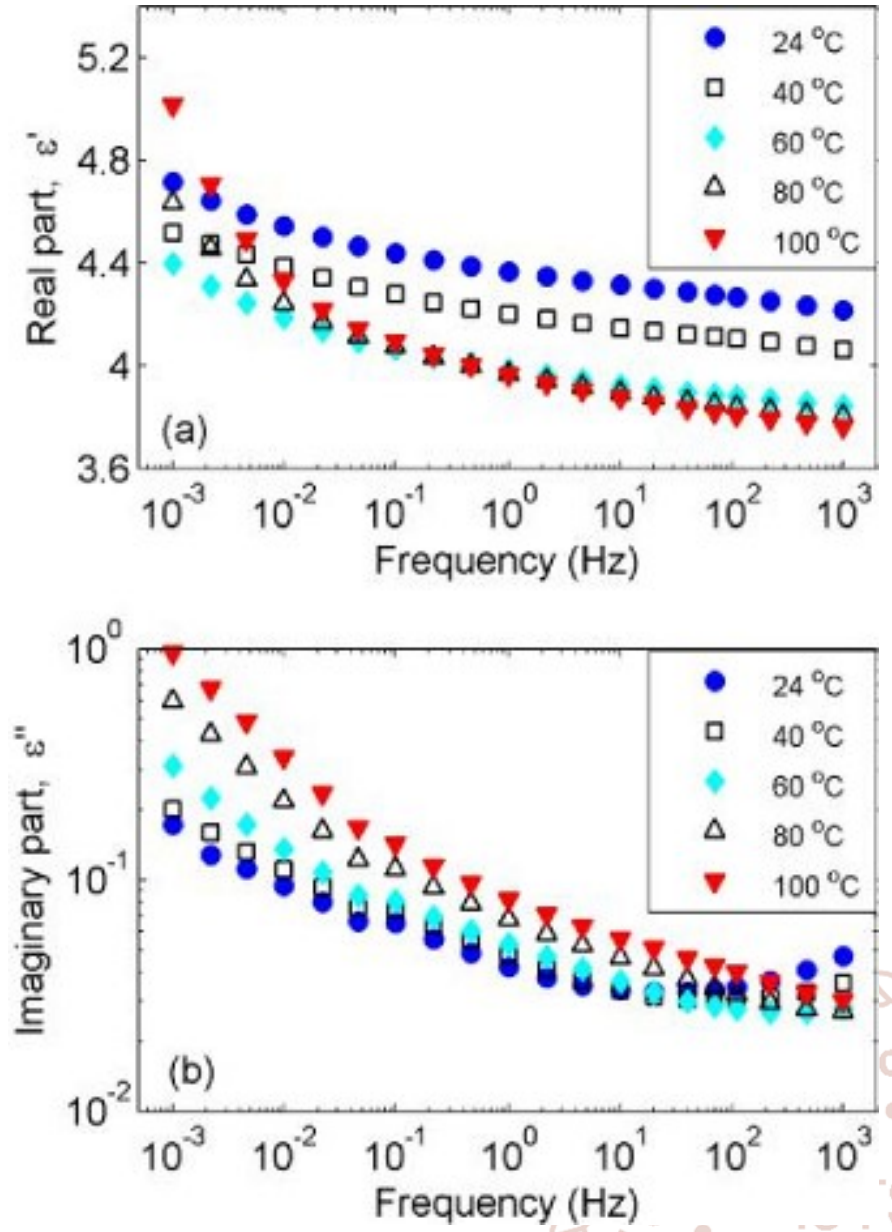

Figure7. Real (a) and imaginary (b) parts of the complex permittivity of filled enamel at different temperatures

\section{Conclusion}

Dielectric properties have been measured and compared for the PD-resistant enamel filled and the conventional insulation. The conventional enamel used in the present study as the reference consisted of a PEI base coat, a PAI overcoat and a modified aromatic PA bonding coat. It is characterized by a dc volume conductivity as low as $610-17$ $\mathrm{S} / \mathrm{m}$. The measured dc volume conductivity of the PAI layer was $\sim 10-15 \mathrm{~S} / \mathrm{m}$, whereas the property of the other two materials was unknown. It was observed that PA layer is relatively more conductive than the PEI and PAI layers, whereas PEI is the most resistive material having dc conductivity at the same order as for conventional enamel coating. On the other hand, filler is much more conductive than the polymeric insulation.

The dielectric properties of filled enamel were experimentally studied in a wide range of temperature and were compared with that of conventional enamel. Increasing temperature resulted in higher dc conductivity and dielectric loss; whereas it yielded lower relative permittivity.
Hence, the additive gave rise to both the real and imaginary parts of the complex permittivity of the filled enamel as compared to those of the conventional one. Both the studied materials, measurements were conducted in a wide range of temperature, showing the increase of dc conductivity and dielectric loss and the decrease of the real part of relative permittivity with temperature.

The performed accelerated aging tests at high temperatures is compared for revealed that the life time of such insulation is substantially increased as compared to both the conventional and the early developed enamel with filler in the top coating only

\section{References}

[1] Bjorklund A, Siberg H. and Paloniemi P, (1995): "Accelerated ageing of a partial discharge resistant enameled round wire [motor winding]," in Proceedings of Electrical Electronics Insulation and Electrical Manufacturing \& Coil Winding Conference, 1995, pp. 417419.

[2] Diaham S and Locatelli M. L, (2013): "Dielectric properties of polyamide-imide," J. Phys. D: Appl. Phys., vol. 46, p. 185302.

[3] Dolui S.K, Pal D. and Maiti S.,(1985) "Synthesis of a Novel Polyesterimide," J. Appl. Polym. Sci., vol. 30, pp. 38673878,

[4] Fabiani D. and Montanari G. C. (2001): "The effect of voltage distortion on ageing acceleration of insulation systems under partial discharge activity," IEEE Electrical Insulation Magazine, vol. 17, pp. 24-33,

[5] Hahn T. A,(1970): "Thermal expansion of copper from 20 to $800 \mathrm{~K}$-Standard reference material 736," Journal of Applied Physics, vol. 41, pp. 5096-5101.

[6] Haq U. S, Jayaram S. H, and Cherney E. A. (2007): "Evaluation of medium voltage enameled wire exposed to fast repetitive voltage pulses," IEEE Transactions on Dielectrics and Electrical Insulation, vol. 14, pp. 194-203.

[7] Hasegawa M, Sakamoto Y., Tanaka Y., and Kobayashi Y.(2010): "Poly(ester imide)s possessing low coefficients of thermal expansion (CTE) and low water absorption (III). Use of bis (4aminophenyl)terephthalate and effect of substituents," European Polymer Journal, vol. 46, pp. 1510-1524,

[8] Ma X., Lee N. H., Oh H. J. , Hwang J. S., and Kim S. J. ,(2010): "Preparation and characterization of silica/polyamide-imide nanocomposite thin films," Nanoscale Res. Lett., vol. 5, pp. 1846-1851.

[9] Paulsson G., Sahlen F., Hillborg H., Björklund A., Takala M., and Andersson J. , (2013): "New type of PD-resistant enameled wire," in 12th International Electrical Insulation Conference Birmingham, UK, , pp. 169-174. 